\title{
Energy and angular momentum flow into a black hole in a binary
}

\author{
Kashif Alvi \\ Physics Division 130-33, California Institute of Technology, Pasadena, California 91125
}

\begin{abstract}
As a black hole in a binary spirals in gradually from large separation, energy and angular momentum flow not only to infinity but also into or out of the hole. In addition, the hole's horizon area increases slowly during this process. In this paper, the changes in the black hole's mass, spin, and horizon area during inspiral are calculated for a hole in a circular binary with a companion body of possibly comparable mass. When the binary is composed of equalmass black holes that have spins aligned with the orbital angular momentum and are rapidly rotating (with spins 99.8 percent of their maximal values), it is found that the fractional increase in the surface area of each hole's horizon is one percent by the time the binary spirals down to a separation $b$ of $6 M$ (where $M$ is the binary's total mass), and seven percent down to $b=2 M$. The flow of energy and angular momentum into the black holes' horizons changes the number of gravitational-wave cycles in the LIGO band by no more than a tenth of a cycle by the time the binary reaches $b=2 M$. The results obtained in this paper are relevant for the detection and analysis of gravitational waves from binary systems containing a black hole.
\end{abstract}

\section{INTRODUCTION}

Binary black holes are expected to be among the primary sources of gravitational waves for interferometric detectors [1]. Since we do not have exact solutions of Einstein's equations 
that represent binary black holes in sufficient generality, we must study these systems perturbatively and/or numerically. One regime in which the evolution of binary black holes is well understood is the early inspiral phase. In this phase, the holes' separation is still much larger than the binary's total mass, and post-Newtonian expansions can be used to analyze the system. Eventually radiation reaction drives the holes together and the post-Newtonian approximation fails. The binary's subsequent evolution must be studied numerically.

While the flow of energy and angular momentum to infinity during inspiral has been calculated to high post-Newtonian order, to date the flow into or out of the black holes' horizons has not been computed except in the extreme-mass-ratio limit; and in that limit, it has been done to very high post-Newtonian order [2] (for numerical work, see e.g. [3]). Absorption (or emission) of energy and angular momentum by the holes' horizons, while much smaller than emission to infinity, might still be important because extraction of weak gravitational signals from noisy detector output using matched filtering requires knowledge of the orbital evolution to very high accuracy, and black hole absorption/emission might affect the evolution at that level. Two purposes of this paper are to calculate black hole absorption/emission of energy and angular momentum to leading order in a circular binary with holes of possibly comparable mass, and to investigate whether it is relevant for detection and analysis of gravitational waves.

A third purpose of this paper is to provide some information on the interface between the inspiral and merger phases of binary evolution. Numerical simulations of binary black holes typically begin computing at this interface and need initial data representing holes that have spiraled in from infinity, i.e. initial data tied to the inspiral phase and to the post-Newtonian expansions used to describe it. One approach to obtaining such initial data is given in [4]. Since initial data of this sort are not yet being used, one needs to relate the masses, spins, and horizon areas of the black holes present in currently used initial data to the corresponding quantities when the holes were infinitely separated. For this purpose, it is necessary to know how these quantities change during inspiral. In this paper, I calculate the leading-order changes in the holes' masses, spins, and horizon areas during inspiral for 
a circular binary.

Recently, Price and Whelan [5] have emphasized the role of angular momentum absorption/emission by rapidly rotating black holes at the end of inspiral, when the holes are beginning to merge. Here I focus on the earlier stages of inspiral, when the holes are widely (or moderately) separated and their gravitational effects on each other can be described using black hole perturbation theory.

The results obtained in this paper are actually valid for a black hole in a binary with any companion body (e.g. a neutron star) that is well separated from the hole. The formulas for the changes in black hole quantities presented here depend only on the companion body's mass and not on its internal structure. These formulas therefore remain valid when the companion is not a black hole.

\section{FRAMEWORK}

I follow the field-theory-in-flat-spacetime notation used in the literature on postNewtonian expansions (e.g. [6,7]) and denote 3-vectors by bold-face letters. A centered dot between 3-vectors denotes the usual inner product in flat 3-space; a hatted 3-vector represents the unit vector in that direction.

Consider a black hole binary undergoing circular motion with the separation $b$ between the holes much larger than their total mass $M=M_{1}+M_{2}$, where $M_{B}$ denotes the mass of the $B$ th hole. Define $\mu=M_{1} M_{2} / M$ and $\eta=\mu / M$. Label the holes BH1 and BH2, and denote their spins by $\mathbf{S}_{B}$ and horizon areas by $A_{B}$ for $B=1,2$. Let $S_{B}=\left(\mathbf{S}_{B} \cdot \mathbf{S}_{B}\right)^{1 / 2}$ be the spin magnitudes, and define the parameters $\chi_{B}$ by $S_{B}=\chi_{B} M_{B}^{2}(B=1,2)$. Throughout this paper I assume $\chi_{B} \leq 0.998(B=1,2)$; this restriction is based on the analysis in [8].

Define each black hole's horizon radius $r_{H B}=M_{B}\left[1+\left(1-\chi_{B}^{2}\right)^{1 / 2}\right]$, angular velocity $\Omega_{H B}=\chi_{B}\left(2 r_{H B}\right)^{-1}$, and surface gravity $\kappa_{B}=\left(1-\chi_{B}^{2}\right)^{1 / 2}\left(2 r_{H B}\right)^{-1}(B=1,2)$. Introduce the following Newtonian quantities for the binary: the orbital angular momentum $\mathbf{L}_{N}$, the orbital angular velocity $\Omega_{N}=\left(M / b^{3}\right)^{1 / 2}$, and the relative velocity $v=(M / b)^{1 / 2}$. By assumption, 
$v \ll 1$.

Since the black holes are widely separated, each hole has a surrounding region that satisfies the following properties: (i) it is far enough from the hole that gravity is weak there; (ii) it does not extend so far that the companion hole's tidal field varies appreciably in the region [9]. We can place in this region an inertial coordinate system in which the hole is (momentarily) at rest. This region and its local coordinates are referred to as the black hole's local asymptotic rest frame (LARF) [9]. Label the two regions around the holes LARF1 and LARF2.

Usually mass and angular momentum are only defined globally in general relativity, using fields at infinity, since precise local definitions are not available. However, for a black hole well separated from its companion, one can define the hole's mass and angular momentum using fields measured in the hole's LARF; these definitions are inherently ambiguous [9 12]. (For further discussion of the ambiguities, see Sec. VI.) I refer to these definitions when discussing a black hole's mass and angular momentum in this paper. I calculate the rates of change of these quantities as measured in the LARF - that is, with respect to time $t$ measured by an inertial observer in the LARF. When integrated over the duration of inspiral, these rates of change should give results exceeding the ambiguities in the definitions of mass and angular momentum, in order to be relevant to the analysis of initial data at the interface between inspiral and merger. This issue will be discussed further in Sec. VI.

I also consider slices of constant time $t$ that begin in the LARF and extend into the black hole, intersecting the horizon in 2-surfaces that correspond to constant ingoing-time slices of a Kerr black hole's horizon. (Alternatively, one can consider slices that intersect a "stretched horizon" as discussed in [13] and references therein.) The rate of area increase of these 2-surfaces can be calculated using the results of Hawking and Hartle [14] combined with black hole perturbation theory [15,16]. The quantities $d M_{B} / d t$ and $d S_{B} / d t$ can then be obtained from $d A_{B} / d t$ using the first law of black hole mechanics $d M=(\kappa / 8 \pi) d A+\Omega_{H} d J$ and the relation $\omega d J=m d M$ for black hole perturbation modes of angular frequency $\omega$ and azimuthal angular number $m[16,17,13]$. (Here $J$ refers to the black hole's angular 
momentum.)

Throughout this paper, I focus on BH1 and the changes in its parameters. The corresponding formulas for BH2 are simply obtained by exchanging the subscripts $1 \leftrightarrow 2$ in the final results [e.g. Eqs. (11)]. In Sec. [II], I consider the special situation in which BH2 is held stationary with respect to BH1. The results from this artificial scenario are used in Sec. IV to analyze a circular binary with black hole spins aligned or anti-aligned with $\mathbf{L}_{N}$. The more general case of spins not fully aligned or anti-aligned with $\mathbf{L}_{N}$ is treated in Sec. $\square$.

\section{STATIONARY COMPANION}

In this section, I calculate the tidal distortion BH1 suffers when $\mathrm{BH} 2$ is held stationary. This involves solving for the Weyl tensor component $\psi_{0}$, which contains complete information about the gravitational perturbation on BH1, using the Teukolsky formalism [15. With $\psi_{0}$ in hand, the rates of change of BH1 parameters can be calculated using the results of Hawking and Hartle [14] and Teukolsky and Press [16].

The first step in this process is to calculate BH2's tidal field as seen in LARF1. I will consider only the lowest-order Newtonian tidal field, which is approximately constant throughout LARF1. To calculate this field and its effect on BH1, consider first a fictitious Euclidean 3-space containing a single stationary body of mass $M_{2}$ at coordinate location $\left(b, \theta_{0}, \phi_{0}\right)$ in a spherical polar coordinate system. The Newtonian gravitational potential at the field point $(r, \theta, \phi)$ is given in these coordinates by

$$
\Phi(r, \theta, \phi)=-4 \pi \frac{M_{2}}{b} \sum_{l=0}^{\infty} \sum_{m=-l}^{l}(2 l+1)^{-1}\left(\frac{r}{b}\right)^{l} Y_{l m}^{*}\left(\theta_{0}, \phi_{0}\right) Y_{l m}(\theta, \phi)
$$

for $r<b$.

We are interested in the gravitational field only in a small neighborhood of the origin satisfying $r \ll b$. In particular, we would like to evaluate the body's tidal field at the origin, so only the $l=2$ part $\Phi^{(2)}$ of $\Phi$ is relevant. The (electric-type) tidal field is given by $\mathcal{E}_{i j}=\Phi_{, i j}^{(2)}$ in Cartesian coordinates. Taking these derivatives in spherical coordinates and 
evaluating in the usual spherical orthonormal basis yields the tidal field components $\mathcal{E}_{\hat{\theta} \hat{\theta}}$, $\mathcal{E}_{\hat{\theta} \hat{\phi}}, \mathcal{E}_{\hat{\phi} \hat{\phi}}$ near the origin $r=0$. The particular combination of relevance to us (see below) is in this way determined to be

$$
\mathcal{E}_{\hat{\phi} \hat{\phi}}-\mathcal{E}_{\hat{\theta} \hat{\theta}}-2 i \mathcal{E}_{\hat{\theta} \hat{\phi}}=\frac{8 \pi \sqrt{6} M_{2}}{5 b^{3}} \sum_{m=-2}^{2}{ }_{2} Y_{2 m}(\theta, \phi) Y_{2 m}^{*}\left(\theta_{0}, \phi_{0}\right) .
$$

Here the functions ${ }_{2} Y_{2 m}(\theta, \phi)$ are spin-weighted spherical harmonics 18$]$.

Return now to the black hole binary. The region near BH1, including LARF1, can be described as a perturbed Kerr black hole, and so can be covered by Boyer-Lindquist coordinates $(t, r, \theta, \phi)$. We would like to solve the Teukolsky equation [15] in this region for the Weyl tensor component $\psi_{0}(r, \theta, \phi)$. If we were considering a single perturbed Kerr black hole as the entire spacetime, the asymptotic form of $\psi_{0}$ as $r / M_{1} \rightarrow \infty$ would be the combination $\mathcal{E}_{\hat{\phi} \hat{\phi}}-\mathcal{E}_{\hat{\theta} \hat{\theta}}-2 i \mathcal{E}_{\hat{\theta} \hat{\phi}}$ of the external tidal field [13], since $\psi_{0}$ vanishes for an unperturbed black hole. In our binary system, $\psi_{0}$ acquires this asymptotic form for $M_{1} \ll r \ll b$, i.e. in LARF1, with the tidal field $\mathcal{E}_{i j}$ being that of BH2. To lowest order, this tidal field is exactly the Newtonian field of a body of mass $M_{2}$ at separation $b$, which was calculated above; in particular, the angular dependence of $\psi_{0}$ in LARF1 is given by Eq. (2), but with $\theta$ and $\phi$ now representing Boyer-Lindquist coordinates, and $\theta_{0}$ and $\phi_{0}$ now representing BH2's angular coordinates as seen in LARF1. Therefore, to solve for the perturbation $\psi_{0}$ on BH1, we impose the LARF1 boundary condition

$$
\psi_{0} \rightarrow \frac{8 \pi \sqrt{6} M_{2}}{5 b^{3}} \sum_{m=-2}^{2}{ }_{2} Y_{2 m}(\theta, \phi) Y_{2 m}^{*}\left(\theta_{0}, \phi_{0}\right)
$$

for $M_{1} \ll r \ll b$.

It now remains to solve the Teukolsky equation for $\psi_{0}$ with the boundary condition (3) and an appropriate no-outgoing-wave boundary condition at the black hole horizon [15]. We express $\psi_{0}$ as a sum of modes

$$
\psi_{0}=\sum_{m=-2}^{2}{ }_{2} Y_{2 m}(\theta, \phi) R_{m}(r)
$$

and solve the radial Teukolsky equation for $R_{m}(r)$ subject to the no-outgoing-wave boundary condition at the horizon. This yields the radial functions (Eq. (5.7) in Ch. VI of [19]) 


$$
R_{m}(r)=C_{m} x^{\gamma_{m}-2}(1+x)^{-\gamma_{m}-2} F\left(-4,1,-1+2 \gamma_{m},-x\right)
$$

for $m \neq 0$. Here

$$
\gamma_{m}=\frac{i m \chi_{1}}{2\left(1-\chi_{1}^{2}\right)^{1 / 2}}, \quad x=\frac{r-r_{H 1}}{2 M_{1}\left(1-\chi_{1}^{2}\right)^{1 / 2}},
$$

and $F$ is a hypergeometric function. The $m=0$ mode can be treated separately; since a full treatment reveals that this mode does not contribute to the rates of change of black hole parameters, I ignore it here. The constants $C_{m}$ are determined by imposing the LARF1 boundary condition (3); we obtain

$$
C_{m}=\frac{8 \pi M_{2}}{5 b^{3} \sqrt{6}} \gamma_{m}\left(\gamma_{m}+1\right)\left(4 \gamma_{m}^{2}-1\right) Y_{2 m}^{*}\left(\theta_{0}, \phi_{0}\right) .
$$

The leading-order tidal distortion of $\mathrm{BH} 1$ due to the presence of a stationary companion of mass $M_{2}$ has now been determined. This information allows us to calculate the rates of change of BH1 quantities using the results of Hawking and Hartle [14]. In fact, given the modal decomposition (4), we can easily obtain the relevant rates using explicit formulas provided by Teukolsky and Press [16]. The results are $d M_{1} / d t=0$ and

$$
\begin{aligned}
& \frac{d A_{1}}{d t}=\frac{64 \pi M_{1}^{5} M_{2}^{2} \chi_{1}^{2} \sin ^{2} \theta_{0}}{5 b^{6}\left(1-\chi_{1}^{2}\right)^{1 / 2}}\left(1-\frac{3}{4} \chi_{1}^{2}+\frac{15}{4} \chi_{1}^{2} \sin ^{2} \theta_{0}\right), \\
& \frac{d S_{1}}{d t}=-\frac{\left(1-\chi_{1}^{2}\right)^{1 / 2}}{8 \pi \chi_{1}} \frac{d A_{1}}{d t}=-\frac{8 M_{1}^{5} M_{2}^{2}}{5 b^{6}} \chi_{1} \sin ^{2} \theta_{0}\left(1-\frac{3}{4} \chi_{1}^{2}+\frac{15}{4} \chi_{1}^{2} \sin ^{2} \theta_{0}\right) .
\end{aligned}
$$

Here $\theta_{0}$ is BH2's $\theta$-coordinate - that is, its polar angle with respect to $\mathbf{S}_{1}$-as measured in LARF1.

Since the effects of only the leading-order tidal field were taken into account above, the expressions (8) are actually valid for any companion body of mass $M_{2}$, not just a black hole. The rates (8) of area increase and spin-down have already been derived by Teukolsky [19] in the extreme-mass-ratio limit, i.e. for $M_{2} \ll M_{1}$. The derivation I have presented above establishes the validity of the expressions (8) for comparable-mass black holes as well. Hartle and collaborators [14, 20, 21, [13] have shown that the spin-down of a black hole by an external tidal field is analogous to the Newtonian tidal friction process in a planet-moon system. 
The results (8) will be used in the next sections to obtain the corresponding formulas for a binary undergoing circular motion.

\section{EQUATORIAL ORBITS}

In this section I study special configurations of the binary in which the black holes are in a circular orbit and have their spins aligned or anti-aligned with the orbital angular momentum $\mathbf{L}_{N}$. In these scenarios there is no precession of the angular momenta: the spins remain aligned or anti-aligned with $\mathbf{L}_{N}$. As a result, the companion to each of the holes orbits in the hole's equatorial plane; more precisely, the external tidal field seen by each of the holes rigidly rotates about an axis parallel or antiparallel to the hole's spin axis.

In Boyer-Lindquist coordinates $(t, r, \theta, \phi)$ centered on BH1, with $\mathbf{S}_{1}$ along $\theta=0$, the $t$ - and $\phi$-dependence of the companion's tidal field enter in the combination $\phi-\Omega t$. The rotation rate $\Omega$ of the tidal field as seen in LARF1 is to leading order $\Omega=\left(\hat{\mathbf{L}}_{N} \cdot \hat{\mathbf{S}}_{1}\right) \Omega_{N}$, where $\hat{\mathbf{L}}_{N} \cdot \hat{\mathbf{S}}_{1}=+1(-1)$ for a prograde (retrograde) orbit. The first correction to this expression for $\Omega$ is $O\left(v^{2}\right)$ higher (see Eq. (3.12) in [ब]), and will be ignored in this paper.

\section{A. Instantaneous rates}

In the rigid $\phi$-rotation case, simple formulas given in Eqs. (7.21) of [13] (and reproduced below) specify the rates of change of black hole quantities in terms of a horizon integral $I$ that depends on the particular perturbing gravitational fields present:

$$
\begin{aligned}
\frac{d S_{1}}{d t} & =\left(\Omega-\Omega_{H 1}\right) I, \quad \frac{d M_{1}}{d t}=\Omega \frac{d S_{1}}{d t}=\Omega\left(\Omega-\Omega_{H 1}\right) I, \\
\frac{\kappa_{1}}{8 \pi} \frac{d A_{1}}{d t} & =\left(\Omega-\Omega_{H 1}\right) \frac{d S_{1}}{d t}=\left(\Omega-\Omega_{H 1}\right)^{2} I .
\end{aligned}
$$

In terms of ingoing Kerr coordinates $(V, r, \theta, \tilde{\phi})$ (see e.g. 22] for a definition), $I$ is an integral of a function of $\theta$ and $(\tilde{\phi}-\Omega V)$ over a constant- $V$ slice of the horizon. Since $\tilde{\phi}$-rotations are isometries of the horizon metric, $I$ is independent of $V$. 
Consider an expansion of $I$ in powers of $M_{1} \Omega$, which is $O\left(v^{3}\right)$ and hence much smaller than 1. The zeroth-order part $I_{0}=\left.I\right|_{\Omega=0}$ is independent of $\Omega$ and, in our situation of binary black holes, can be easily obtained from the results for a stationary companion. From Eqs. (9), we have $\left.\dot{S}_{1}\right|_{\Omega=0}=-\Omega_{H 1} I_{0}$, where an overdot indicates a time derivative. But $\Omega=0$ corresponds to a stationary companion, and in this case we have an explicit expression for $\dot{S}_{1}$ in Eqs. (8). Equating $\dot{S}_{1}$ in Eqs. (8) to $-\Omega_{H 1} I_{0}$ yields

$$
I_{0}\left(\theta_{0}\right)=\frac{16 r_{H 1}}{5 b^{6}} M_{1}^{5} M_{2}^{2} \sin ^{2} \theta_{0}\left(1-\frac{3}{4} \chi_{1}^{2}+\frac{15}{4} \chi_{1}^{2} \sin ^{2} \theta_{0}\right),
$$

where $\theta_{0}=\pi / 2$ for the equatorial orbits considered in this section. The general expression (10) with a wider range of values for $\theta_{0}$ will be used for non-equatorial orbits in the next section. Since the first correction to $I_{0}$ in the expansion of $I$ in powers of $M_{1} \Omega$ is $O\left(M_{1} \Omega\right)=O\left(v^{3}\right)$, I will approximate $I$ by $I_{0}$ throughout this paper.

Note that Eqs. (9) are, strictly speaking, valid only for constant rotation rates $\Omega$. In our situation, radiation reaction drives the binary together and so $\Omega$ changes during inspiral. However, the timescale for these changes is the inspiral timescale $\tau_{\text {ins }} \sim b v^{-6}$, where " $\sim$ " means "is of the order of"; this is to be compared to the timescale $\kappa_{1}^{-1}$ on which the divergence and shear of the null generators of the horizon probe the future [14,[17, [13]. By assumption, $\chi_{1}$ is less than or equal to 0.998 ; this implies $\kappa_{1}^{-1}<34 M_{1}$, so $\kappa_{1}^{-1}$ is much smaller than $\tau_{\text {ins }}$. Therefore Eqs. (9) are valid in our binary system to a very good approximation. The various timescales of interest to us will be discussed in more detail below.

Note also that Eqs. (9) [and, in addition, Eqs. (11), (21), and (22) below] are valid only when integrated over time intervals much longer than $\kappa_{1}^{-1}$ (see the discussion in Sec. VI.C.11 of [13]). In this paper, I am interested in integrating these equations over the entire inspiralthat is, over time intervals of order $\tau_{\text {ins }}$-so this condition is certainly satisfied.

After putting $I_{0}(\pi / 2)$ and $\Omega=\left(\hat{\mathbf{L}}_{N} \cdot \hat{\mathbf{S}}_{1}\right) \Omega_{N}$ into Eqs. (9), we obtain the following rates of change of BH1 quantities for a circular orbit with spins aligned or anti-aligned with $\mathbf{L}_{N}$ :

$$
\frac{d S_{1}}{d t}=\left(\Omega-\Omega_{H 1}\right) I_{0}(\pi / 2)
$$




$$
\begin{aligned}
& =\left(\frac{d J}{d t}\right)_{N} \frac{v^{5}}{4}\left(\frac{M_{1}}{M}\right)^{3}\left(1+3 \chi_{1}^{2}\right)\left\{-\chi_{1}+2\left(\hat{\mathbf{L}}_{N} \cdot \hat{\mathbf{S}}_{1}\right)\left[1+\left(1-\chi_{1}^{2}\right)^{1 / 2}\right] \frac{M_{1}}{M} v^{3}\right\}, \\
\frac{d M_{1}}{d t} & =\Omega\left(\Omega-\Omega_{H 1}\right) I_{0}(\pi / 2) \\
& =\left(\frac{d E}{d t}\right)_{N} \frac{v^{5}}{4}\left(\frac{M_{1}}{M}\right)^{3}\left(1+3 \chi_{1}^{2}\right)\left\{-\left(\hat{\mathbf{L}}_{N} \cdot \hat{\mathbf{S}}_{1}\right) \chi_{1}+2\left[1+\left(1-\chi_{1}^{2}\right)^{1 / 2}\right] \frac{M_{1}}{M} v^{3}\right\}, \\
\frac{d A_{1}}{d t} & =8 \pi \kappa_{1}^{-1}\left(\Omega-\Omega_{H 1}\right)^{2} I_{0}(\pi / 2) \\
& =\frac{64 \pi M_{1}^{5} M_{2}^{2}\left(1+3 \chi_{1}^{2}\right)}{5 b^{6}\left(1-\chi_{1}^{2}\right)^{1 / 2}}\left\{\chi_{1}-2\left(\hat{\mathbf{L}}_{N} \cdot \hat{\mathbf{S}}_{1}\right)\left[1+\left(1-\chi_{1}^{2}\right)^{1 / 2}\right] \frac{M_{1}}{M} v^{3}\right\}^{2} .
\end{aligned}
$$

In these formulas, the Newtonian quadrupole expressions for energy and angular momentum flow to infinity are [23,24]

$$
\left(\frac{d E}{d t}\right)_{N}=\frac{32}{5} \eta^{2} v^{10}, \quad\left(\frac{d J}{d t}\right)_{N}=\frac{32}{5} \eta^{2} M v^{7},
$$

where $v=(M / b)^{1 / 2}$ and $\eta=M_{1} M_{2} / M^{2}$. Note that energy and angular momentum absorption/emission by a rotating (non-rotating) black hole is 2.5 (4) post-Newtonian orders below the quadrupole emission (12) to infinity, as first derived in the extreme-mass-ratio limit by Poisson and Sasaki [25] and Tagoshi, Mano, and Takasugi [2]. The rates of change for BH2 are obtained by exchanging the subscripts $1 \leftrightarrow 2$ in the formulas (11).

The energy absorption/emission rate $\dot{M}_{1}$ given above agrees in the limit $M_{2} / M \rightarrow 0$ with the lowest-order expression obtained by Tagoshi, Mano, and Takasugi [2]. Those authors have calculated this rate in the extreme-mass-ratio limit, for a circular equatorial orbit, to much higher order in $v$ than I have done here. However, their results are not applicable to comparable-mass binaries, while the formulas (11) are.

The expressions (11) are valid even if BH1's companion is not a black hole, provided the companion's mass is substituted for $M_{2}$.

\section{B. Total changes during inspiral}

In this subsection, I integrate Eqs. (11) to calculate the total changes in $M_{1}, S_{1}$, and $A_{1}$ during inspiral. I take into account only the leading-order Newtonian effects of radiation 
reaction when computing orbital decay; given this approximation, the orbital separation $b$ evolves as 24,22

$$
b(t)=b_{0}\left(1-t / \tau_{0}\right)^{1 / 4}
$$

where $\tau_{0}=(5 / 256) b_{0}^{4}\left(\mu M^{2}\right)^{-1}$. I also ignore all post-Newtonian corrections to the orbital angular velocity $\Omega_{N}$.

It is convenient to parametrize the orbit by separation $b$ instead of time $t$. The total change in a parameter, say $S_{1}$, from infinite separation to separation $b$ is denoted $\Delta S_{1}(b)$ and is calculated by integrating Eqs. (11). As a first approximation, the quantities $M_{B}$ and $S_{B}(B=1,2)$ on the right-hand sides of Eqs. (11) can be considered constants during inspiral. The reason is that the timescales for evolution of $M_{B}$ and $S_{B}$ are much longer than the inspiral timescale $\tau_{\text {ins }} \sim b v^{-6}$. Indeed, the timescale for evolution of the masses is $\tau_{M} \sim M_{B} / \dot{M}_{B} \sim b v^{-13}$, and for the spins is $\tau_{S} \sim S_{B} / \dot{S}_{B} \sim b v^{-10}$. So $\tau_{M} \gg \tau_{S} \gg \tau_{\text {ins }}$ and we can safely treat $M_{B}$ and $S_{B}(B=1,2)$ as constants on the right-hand sides of Eqs. (11) when integrating over inspiral.

With these approximations, the normalized changes in $\mathrm{BH} 1$ parameters from infinite separation to separation $b$ are

$$
\begin{aligned}
\frac{\Delta S_{1}}{M_{1}^{2}}(b)= & \frac{\eta M_{1}}{4 M}\left(1+3 \chi_{1}^{2}\right)\left\{-\frac{\chi_{1}}{4}\left(\frac{M}{b}\right)^{2}+\left(\hat{\mathbf{L}}_{N} \cdot \hat{\mathbf{S}}_{1}\right)\left[1+\left(1-\chi_{1}^{2}\right)^{1 / 2}\right] \frac{2 M_{1}}{7 M}\left(\frac{M}{b}\right)^{7 / 2}\right\} \\
\frac{\Delta M_{1}}{M_{1}}(b)= & \frac{\eta}{4}\left(\frac{M_{1}}{M}\right)^{2}\left(1+3 \chi_{1}^{2}\right)\left\{-\left(\hat{\mathbf{L}}_{N} \cdot \hat{\mathbf{S}}_{1}\right) \frac{\chi_{1}}{7}\left(\frac{M}{b}\right)^{7 / 2}+\left[1+\left(1-\chi_{1}^{2}\right)^{1 / 2}\right] \frac{M_{1}}{5 M}\left(\frac{M}{b}\right)^{5}\right\} \\
\frac{\Delta A_{1}}{A_{1}}(b)= & \frac{\eta M_{1}^{2}\left(1+3 \chi_{1}^{2}\right)}{8 M r_{H 1}\left(1-\chi_{1}^{2}\right)^{1 / 2}}\left[\frac{\chi_{1}^{2}}{2}\left(\frac{M}{b}\right)^{2}-\left(\hat{\mathbf{L}}_{N} \cdot \hat{\mathbf{S}}_{1}\right) \frac{8 \chi_{1}}{7} \frac{r_{H 1}}{M}\left(\frac{M}{b}\right)^{7 / 2}\right. \\
& \left.+\frac{4}{5}\left(\frac{r_{H 1}}{M}\right)^{2}\left(\frac{M}{b}\right)^{5}\right],
\end{aligned}
$$

where $r_{H 1}=M_{1}\left[1+\left(1-\chi_{1}^{2}\right)^{1 / 2}\right]$. To evaluate these changes, one can put into the formulas (14) the values of $M_{1}, S_{1}$, and $A_{1}$ at infinite separation or, for that matter, at any separation much larger than $M$, because the changes in these quantities during inspiral are small. 


\begin{tabular}{ccccc}
\hline \hline$\chi_{1}$ & $b / M=100$ & $b / M=20$ & $b / M=6$ & $b / M=2$ \\
\hline 0 & $9 . \times 10^{-10}$ & $2 . \times 10^{-7}$ & $2 . \times 10^{-5}$ & \\
0.5 & $-7 . \times 10^{-7}$ & $-2 . \times 10^{-5}$ & $-2 . \times 10^{-4}$ & \\
0.998 & $-3 . \times 10^{-6}$ & $-8 . \times 10^{-5}$ & $-8 . \times 10^{-4}$ & $-6 . \times 10^{-3}$ \\
\hline \hline
\end{tabular}

TABLE I. Normalized change $\Delta S_{1} / M_{1}^{2}$ in spin evaluated at $b / M=100,20$, and 6 for an equal-mass binary with $\hat{\mathbf{L}}_{N} \cdot \hat{\mathbf{S}}_{1}=1$. For rapidly rotating holes $\left(\chi_{1}=\chi_{2}=0.998\right)$, this change is also evaluated at $b / M=2$.

Once again, the changes for $\mathrm{BH} 2$ are obtained by exchanging the subscripts $1 \leftrightarrow 2$ in the expressions (14).

The normalized parameter changes (14), evaluated at different stages during inspiral, are displayed in Tables 此四 for an equal-mass binary $\left(M_{1}=M_{2}\right)$ with $\hat{\mathbf{L}}_{N} \cdot \hat{\mathbf{S}}_{1}=1$. Since a binary composed of slowly rotating black holes is expected to be undergoing a transition from inspiral to merger by the time it reaches $b=6 M$, the endpoint of integration is chosen to be $b=6 M$ when $\chi_{1}=0$ and 0.5 . For rapidly rotating holes $\left(\chi_{1}=\chi_{2}=0.998\right)$, the endpoint is chosen to be $b=2 M$. The assumption $M \ll b$ is not valid at and near these endpoints. The results presented here are most accurate in the early stages of inspiral, when the black holes are widely separated, and are a rough estimate of the true parameter changes in the late stages of inspiral.

\section{Effect on orbital evolution}

The orbital evolution of binary black holes is affected by the absorption/emission of energy and angular momentum by the holes. In particular, the number of orbits - and hence the number of gravitational-wave cycles emitted to infinity - changes when black hole absorption/emission is accounted for. To estimate this effect, let us consider a circular, nearly Newtonian binary, with spins aligned or anti-aligned with $\mathbf{L}_{N}$, that is losing orbital energy 


\begin{tabular}{ccccc}
\hline \hline$\chi_{1}$ & $b / M=100$ & $b / M=20$ & $b / M=6$ & $b / M=2$ \\
\hline 0 & $3 . \times 10^{-13}$ & $1 . \times 10^{-9}$ & $4 . \times 10^{-7}$ & \\
0.5 & $-2 . \times 10^{-10}$ & $-5 . \times 10^{-8}$ & $-3 . \times 10^{-6}$ & \\
0.998 & $-9 . \times 10^{-10}$ & $-2 . \times 10^{-7}$ & $-2 . \times 10^{-5}$ & $-6 . \times 10^{-4}$ \\
\hline \hline
\end{tabular}

TABLE II. Normalized change $\Delta M_{1} / M_{1}$ in mass evaluated at $b / M=100,20$, and 6 for an equal-mass binary with $\hat{\mathbf{L}}_{N} \cdot \hat{\mathbf{S}}_{1}=1$. For rapidly rotating holes $\left(\chi_{1}=\chi_{2}=0.998\right)$, this change is also evaluated at $b / M=2$.

\begin{tabular}{ccccc}
\hline \hline$\chi_{1}$ & $b / M=100$ & $b / M=20$ & $b / M=6$ & $b / M=2$ \\
\hline 0 & $6 . \times 10^{-13}$ & $2 . \times 10^{-9}$ & $8 . \times 10^{-7}$ & \\
0.5 & $2 . \times 10^{-7}$ & $5 . \times 10^{-6}$ & $4 . \times 10^{-5}$ & \\
0.998 & $5 . \times 10^{-5}$ & $1 . \times 10^{-3}$ & $1 . \times 10^{-2}$ & $7 . \times 10^{-2}$ \\
\hline \hline
\end{tabular}

TABLE III. Normalized change $\Delta A_{1} / A_{1}$ in horizon area evaluated at $b / M=100,20$, and 6 for an equal-mass binary with $\hat{\mathbf{L}}_{N} \cdot \hat{\mathbf{S}}_{1}=1$. For rapidly rotating holes $\left(\chi_{1}=\chi_{2}=0.998\right)$, this change is also evaluated at $b / M=2$. 
and angular momentum to infinity via Newtonian quadrupole radiation (12), and to the black holes via tidal interaction as specified by Eqs. (11). Since $\dot{M}_{B}=\Omega \dot{S}_{B}=\left(\hat{\mathbf{L}}_{N} \cdot \hat{\mathbf{S}}_{B}\right) \Omega_{N} \dot{S}_{B}$ ( $B=1,2)$, circular, nearly Newtonian orbits remain circular. Therefore the evolution of the separation $b(t)$ is determined by setting the rate of change of Newtonian orbital energy (given by $\left.E_{\text {orb }}=-M_{1} M_{2} / 2 b\right)$ to the rate of energy loss to infinity and to the holes:

$$
\frac{d E_{\mathrm{orb}}}{d t}=\frac{M_{1} M_{2}}{2 b^{2}} \frac{d b}{d t}=-\left(\frac{d E}{d t}\right)_{N}-\frac{d M_{1}}{d t}-\frac{d M_{2}}{d t}
$$

where $(d E / d t)_{N}$ is given in Eqs. (12) and $\dot{M}_{1}$ in Eqs. (11) [with $\dot{M}_{2}$ obtained by exchanging the subscripts $1 \leftrightarrow 2$ in Eqs. (11)].

The number of gravitational-wave cycles $N_{1}$ emitted to infinity from initial time $t_{i}$ to final time $t_{f}$ (corresponding to separations $b_{i}$ and $b_{f}$ ) is given by

$$
N=\int_{t_{i}}^{t_{f}} d t \frac{\Omega_{N}}{\pi}=\frac{1}{\pi} \int_{b_{i}}^{b_{f}} d b \frac{d t}{d b}\left(\frac{M}{b^{3}}\right)^{1 / 2},
$$

where $d t / d b$ is determined from Eq. (15). This number is to be compared with the number of cycles $N_{2}$ obtained by ignoring black hole absorption/emission of energy and angular momentum, i.e. by setting $\dot{E}_{\text {orb }}$ equal to $-(d E / d t)_{N}$. The difference $\Delta N=N_{1}-N_{2}$ measures the effect of black hole absorption/emission on the binary's orbital evolution.

The values of $\Delta N$ obtained by setting $b_{i}$ to be the separation at which the gravitationalwave frequency is $10 \mathrm{~Hz}$ (the low-frequency end of the LIGO band), $\chi_{1}$ and $\chi_{2}$ to be 0.998 , and the spins to be aligned with $\mathbf{L}_{N}$ (i.e. $\hat{\mathbf{L}}_{N} \cdot \hat{\mathbf{S}}_{1}=\hat{\mathbf{L}}_{N} \cdot \hat{\mathbf{S}}_{2}=1$ ) are displayed in Table IV for various choices of total mass $M$ (in units of a solar mass $M_{\odot}$ ) and mass ratio $M_{1} / M_{2}$. In the table, the numbers without parentheses are obtained by setting $b_{f}=6 M$, and those with parentheses by setting $b_{f}$ to be the larger of $2 M$ or the separation at which the wave frequency is $1000 \mathrm{~Hz}$ (the high-frequency end of the LIGO band). For non-rotating black holes $\left(\chi_{1}=\chi_{2}=0\right)$, the corresponding values of $\Delta N$ (with $b_{f}=6 M$ ) are all less than $10^{-2}$.

The values of $\Delta N$ in Table $\mathbb{\square}$ indicate that black hole absorption/emission of energy and angular momentum during inspiral may not be an important effect for the detection (by LIGO and VIRGO) and analysis of gravitational waves from comparable-mass black 


\begin{tabular}{cccc}
\hline \hline$M\left(M_{\odot}\right)$ & $M_{1} / M_{2}=1$ & $M_{1} / M_{2}=2$ & $M_{1} / M_{2}=4$ \\
\hline 5 & $0.07(0.07)$ & $0.11(0.11)$ & $0.23(0.24)$ \\
20 & $0.05(0.07)$ & $0.07(0.10)$ & $0.16(0.22)$ \\
50 & $0.03(0.06)$ & $0.05(0.08)$ & $0.11(0.18)$ \\
\hline \hline
\end{tabular}

TABLE IV. Change $\Delta N$ in the number of gravitational-wave cycles due to black hole absorption/emission, for various values of total mass $M$ and mass ratio $M_{1} / M_{2}$. The initial separation is such that the wave frequency is $10 \mathrm{~Hz}$ and the spins satisfy $\chi_{1}=\chi_{2}=0.998$ and $\hat{\mathbf{L}}_{N} \cdot \hat{\mathbf{S}}_{1}=\hat{\mathbf{L}}_{N} \cdot \hat{\mathbf{S}}_{2}=1$. The numbers without parentheses are for a final separation $b_{f}$ of $6 M$; those with parentheses are for $b_{f}$ equal to the larger of $2 M$ or the separation at which the wave frequency is $1000 \mathrm{~Hz}$.

holes. Indeed, post-Newtonian corrections to the equations of motion and energy loss have far greater influence on the number of wave cycles emitted by the binary [6,7]. It should be noted, however, that black hole absorption/emission could have a much larger impact on the orbital evolution of rapidly rotating holes when they are beginning to merge, as suggested by Price and Whelan [5]. They have presented models in which the tidal torque that results from black hole absorption/emission of angular momentum plays a crucial role in the late stages of binary evolution (see Fig. 1 in [5]). The perturbative methods used in this paper (based on wide separation of the binary) are not valid in the close limit analyzed in [5].

It has been pointed out by Hughes [3] that in the extreme-mass-ratio limit, black hole absorption/emission can strongly influence the binary's orbital evolution and is an important effect for LISA.

\section{NON-EQUATORIAL ORBITS}

In general, binary black holes are not expected to have spins aligned with the orbital angular momentum $\mathbf{L}_{N}$. This misalignment causes the spins and orbit to precess in a com- 
plicated way due to spin-orbit and spin-spin coupling [26,7]. Each black hole's companion is not in general confined to the hole's equatorial plane, and so the formulas in the previous section are not applicable. However, for orbits suitably close to the equatorial plane (see below for details), one can imagine using an approximation scheme in which at each instant the companion's $\theta$-velocity is ignored; that is, the companion is taken to be rigidly rotating in the $\phi$-direction at each point on the orbit. The changes in black hole parameters can then be calculated by putting the instantaneous $\phi$-velocity into the rigid $\phi$-rotation formulas (9) at each point on the orbit. In this section, I construct such an approximation scheme.

\section{A. Description of orbit}

The evolutions of the spins and orbit are described by the equations [26, []

$$
\dot{\mathbf{S}}_{B}=\mathbf{\Omega}_{\text {spin }}^{(B)} \times \mathbf{S}_{B}, \quad \dot{\mathbf{L}}_{N}=\mathbf{\Omega}_{\text {orb }} \times \mathbf{L}_{N}-\frac{32}{5} \eta^{2} M v^{7} \hat{\mathbf{L}}_{N}
$$

for $B=1,2$. The orders of magnitude of the precession frequencies are $\Omega_{\text {spin }} \sim v^{3} b^{-1}$ and $\Omega_{\text {orb }} \sim v^{4} b^{-1}$. Since the Newtonian angular velocity is $\Omega_{N} \sim v b^{-1}$, both $\Omega_{\text {spin }}$ and $\Omega_{\text {orb }}$ are much smaller than $\Omega_{N}$. This means that, over a few orbital periods, $\mathbf{L}_{N}(t)$ and $\mathbf{S}_{B}(t)$ do not change much due to precession. Thus, the companion's orbit as seen in LARF1 is to a good approximation confined to a single plane with normal vector $\mathbf{n}=\hat{\mathbf{L}}_{N}(t)$ along the instantaneous direction of the orbital angular momentum, on timescales of a few orbital periods.

In this subsection, I analyze the trajectory of a particle in a planar, circular orbit of arbitrary orientation in a fictitious Euclidean 3-space in terms of spherical coordinates. This information will be used to specify the rotation rate and orientation of the companion's tidal field as seen in LARF1. Denote the particle's radial coordinate by $b$, its constant (nonnegative) angular velocity by $\omega$, and the normal to its orbital plane by $\mathbf{n}$. The angle of inclination of the normal with respect to the $z$-axis is denoted $\theta_{n}$; so $\cos \theta_{n}=\mathbf{n} \cdot \mathbf{e}_{\hat{z}}=n_{z}$. Assume the orbit is centered on the origin, so the particle's position $\mathbf{X}(t)$ at time $t$ is given by a rotation $R(\mathbf{n}, \omega t)$ about $\mathbf{n}$, by an angle $\omega t$, of the initial position $\mathbf{X}_{0}$. 
In Cartesian coordinates, the particle's trajectory is given by $\mathbf{X}(t)=\mathbf{X}_{0} \cos \omega t+$ $\left(\mathbf{n} \times \mathbf{X}_{0}\right) \sin \omega t$. In terms of the particle's angular coordinates $\theta(t)$ and $\phi(t), \mathbf{X}(t)$ is equal to $b[\sin \theta(t) \cos \phi(t), \sin \theta(t) \sin \phi(t), \cos \theta(t)]$. I choose the initial position to be in the equatorial plane, i.e. $Z_{0}=\mathbf{X}_{0} \cdot \mathbf{e}_{\hat{z}}=0$. This choice does not affect the orbit-averaged quantities I calculate later in this section.

The angular functions $\theta(t)$ and $\phi(t)$ can now be expressed in terms of $\mathbf{n}$ and $\omega$ using the

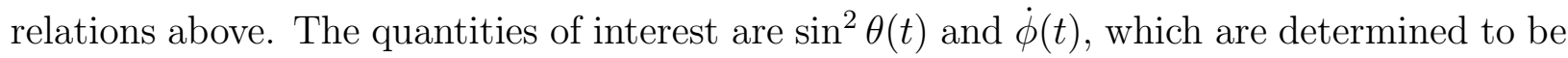

$$
\sin ^{2} \theta(t)=1-\sin ^{2} \theta_{n} \sin ^{2} \omega t, \quad \dot{\phi}(t)=\frac{\omega \cos \theta_{n}}{\sin ^{2} \theta(t)} .
$$

\section{B. Approximation scheme}

Return now to our black hole binary, and go to Boyer-Lindquist coordinates $(t, r, \theta, \phi)$ in LARF1. The companion's trajectory as seen in LARF1 will be described (to lowest order in $v$ ) by angular functions $\theta(t)$ and $\phi(t)$ given by the expressions (18) with $\omega$ replaced by $\Omega_{N}$ and $\theta_{n}$ now referring to the angle of inclination of $\mathbf{L}_{N}(t)$ with respect to $\mathbf{S}_{1}(t)$, that is, $\cos \theta_{n}=\hat{\mathbf{L}}_{N}(t) \cdot \hat{\mathbf{S}}_{1}(t)$. After these substitutions, we have

$$
\sin ^{2} \theta(t)=1-\left(1-\left[\hat{\mathbf{L}}_{N}(t) \cdot \hat{\mathbf{S}}_{1}(t)\right]^{2}\right) \sin ^{2} \Omega_{N} t, \quad \dot{\phi}(t)=\frac{\Omega_{N} \hat{\mathbf{L}}_{N}(t) \cdot \hat{\mathbf{S}}_{1}(t)}{\sin ^{2} \theta(t)} .
$$

Since $\theta_{n}$ is now time dependent, these expressions are meaningful only when used to calculate orbit-averaged quantities.

Consider the regime in which $\sin ^{2} \theta(t)$ and $\dot{\phi}(t)$ are slowly varying; more precisely, require them to be approximately constant on the timescale $\kappa_{1}^{-1}$ associated with the horizon. As noted before, this is the timescale on which the null generators of the horizon probe the future [14, 17, 13]. The teleological behavior of the horizon is, however, exponentially limited; that is, the influence of future events on the horizon decays exponentially in time, with decay rate $\kappa_{1}$ (see, e.g., the discussion of teleological Green functions in [13]). We thus require $|\dot{\phi} / \ddot{\phi}|$ and $\left|\left(1 / \sin ^{2} \theta\right) d\left(\sin ^{2} \theta\right) / d t\right|^{-1}$ [which are the same to leading order by Eqs. (19)] to be only several times larger than $\kappa_{1}^{-1}$, rather than orders of magnitude larger. 
By assumption, $\chi_{1} \leq 0.998$, so $\kappa_{1}^{-1}$ is less than $34 M_{1}$. Our requirement can then be expressed as

$$
34 \alpha M_{1} \Omega_{N} \leq \frac{\sin ^{2} \theta(t)}{\sin ^{2} \theta_{n}\left|\sin 2 \Omega_{N} t\right|}
$$

for all $t$, where $\alpha$ is a number roughly in the range 2-4. A sufficient condition for this constraint to be satisfied is $\cot ^{2} \theta_{n} \geq 34 \alpha M_{1} \Omega_{N}$. This requires $\hat{\mathbf{L}}_{N}(t)$ to be near one of the polar axes $\pm \hat{\mathbf{S}}_{1}(t)$, which correspond to $\theta=0, \pi$; or, equivalently, the orbital plane must be near the equatorial plane.

We are interested in separations as small as $b=6 M$, so $\Omega_{N}$ can be as large as $\left(6^{3 / 2} M\right)^{-1}$. For this reason, I impose the constraint $\cot ^{2} \theta_{n} \geq 34 \alpha 6^{-3 / 2}$ and set $\alpha$ to be approximately 3, obtaining the approximate constraints $0 \leq \theta_{n} \lesssim \pi / 9$ or $8 \pi / 9 \lesssim \theta_{n} \leq \pi$. In other words, $\hat{\mathbf{L}}_{N}(t)$ is within 20-degree cones around the polar axes, or, equivalently, the inclination angle of the orbit with respect to the equatorial plane is less than or (approx.) equal to 20 degrees.

For the approximation scheme in this section to be valid, we require further that in the horizon's reference frame, the external tidal field should rotate primarily in the $\phi$-direction and not significantly in the $\theta$-direction. More precisely, we require $|\dot{\theta}| \ll\left|\dot{\phi}-\Omega_{H 1}\right|$. The rates of change presented in Eqs. (21) and (22) below are subject to this condition. For most values of $\chi_{1}$, this condition is automatically satisfied throughout inspiral (down to $\left.b=6 M\right)$. Even if it is not satisfied at some point during inspiral, the restriction on $\theta_{n}$ discussed above ensures that the effect of the $\theta$-motion, when integrated over inspiral, is negligible compared to that of the $\phi$-motion, for almost all values of $\chi_{1}$.

With the above restriction on $\theta_{n}$, we can at each instant take $\sin ^{2} \theta(t)$ and $\dot{\phi}(t)$ to be constant relative to the horizon timescale $\kappa_{1}^{-1}$, and apply the rigid $\phi$-rotation formulas (9) with the instantaneous values $\theta(t)$ and $\dot{\phi}(t)$ put in. This yields

$$
\begin{array}{rlr}
\frac{d S_{1}}{d t} & =\left[\dot{\phi}(t)-\Omega_{H 1}\right] I_{0}[\theta(t)], \quad \frac{d M_{1}}{d t}=\dot{\phi}(t)\left[\dot{\phi}(t)-\Omega_{H 1}\right] I_{0}[\theta(t)], \\
\frac{\kappa_{1}}{8 \pi} \frac{d A_{1}}{d t} & =\left[\dot{\phi}(t)-\Omega_{H 1}\right]^{2} I_{0}[\theta(t)],
\end{array}
$$


where $\theta(t)$ and $\dot{\phi}(t)$ are given by Eqs. (19) and $I_{0}$ by Eq. (10).

\section{Orbit-averaged quantities}

Next I would like to average these rates of change over an orbit assuming the binary's masses, spins, separation, and orbital angular momentum are approximately constant over an orbital period. This assumption is justified by the following ordering of the relevant timescales: $\Omega_{N}^{-1} \ll \Omega_{\text {spin }}^{-1}, \Omega_{\text {orb }}^{-1} \ll \tau_{\text {ins }} \ll \tau_{S} \ll \tau_{M}$. We can therefore take all the quantities on the right-hand sides of Eqs. (21) except $\theta(t)$ and $\dot{\phi}(t)$ to be constant, to a good approximation, when averaging over an orbit. Denote orbit averages by angular brackets \langle\rangle ; so,

for example, $\left\langle\dot{S}_{1}\right\rangle=\left(\Omega_{N} / 2 \pi\right) \int_{0}^{2 \pi / \Omega_{N}} \dot{S}_{1} d t$. Plugging the expressions (19) into Eqs. (21) and performing the orbit averages (as defined above) yields

$$
\begin{aligned}
\left\langle\frac{d S_{1}}{d t}\right\rangle= & \frac{r_{H 1}}{10 b^{6}} M_{1}^{5} M_{2}^{2}\left(16\left(1+3 \chi_{1}^{2}\right)\left\{2 \Omega_{N} \mathcal{N}_{1}(t)-\Omega_{H 1}\left[\mathcal{N}_{1}^{2}(t)+1\right]\right\}\right. \\
& \left.+15 \chi_{1}^{2}\left[\mathcal{N}_{1}^{2}(t)-1\right]\left\{4 \Omega_{N} \mathcal{N}_{1}(t)-\Omega_{H 1}\left[3 \mathcal{N}_{1}^{2}(t)+1\right]\right\}\right) \\
\left\langle\frac{d M_{1}}{d t}\right\rangle= & \frac{2 r_{H 1}}{5 b^{6}} M_{1}^{5} M_{2}^{2} \Omega_{N} \mathcal{N}_{1}(t)\left(2 \Omega_{N}\left(4-3 \chi_{1}^{2}\right) \operatorname{sign}\left[\mathcal{N}_{1}(t)\right]-8 \Omega_{H 1}\left(1+3 \chi_{1}^{2}\right)\right. \\
& \left.+15 \chi_{1}^{2}\left\{2 \Omega_{N} \mathcal{N}_{1}(t)+\Omega_{H 1}\left[1-\mathcal{N}_{1}^{2}(t)\right]\right\}\right) \\
\left\langle\frac{d A_{1}}{d t}\right\rangle= & \frac{8 \pi r_{H 1}^{2} M_{1}^{5} M_{2}^{2}}{5 b^{6}\left(1-\chi_{1}^{2}\right)^{1 / 2}}\left(( 1 6 - 1 2 \chi _ { 1 } ^ { 2 } ) \left\{\Omega_{H 1}^{2}\left[\mathcal{N}_{1}^{2}(t)+1\right]-4 \Omega_{H 1} \Omega_{N} \mathcal{N}_{1}(t)\right.\right. \\
& \left.+2 \Omega_{N}^{2}\left|\mathcal{N}_{1}(t)\right|\right\}+15 \chi_{1}^{2}\left\{\Omega_{H 1}^{2}\left[3 \mathcal{N}_{1}^{4}(t)+2 \mathcal{N}_{1}^{2}(t)+3\right]\right. \\
& \left.\left.-8 \Omega_{H 1} \Omega_{N} \mathcal{N}_{1}(t)\left[\mathcal{N}_{1}^{2}(t)+1\right]+8 \Omega_{N}^{2} \mathcal{N}_{1}^{2}(t)\right\}\right)
\end{aligned}
$$

where $\mathcal{N}_{B}(t)=\hat{\mathbf{L}}_{N}(t) \cdot \hat{\mathbf{S}}_{B}(t)$ for $B=1,2$. The corresponding expressions for BH2 can be obtained by exchanging the subscripts $1 \leftrightarrow 2$ in Eqs. (22). Note that these equations are valid only for $\mathcal{N}_{B}(t)$ suitably close to \pm 1 , as discussed above. The formulas (22) can be applied to a black hole in a binary with any companion body (e.g. a neutron star) that has mass $M_{2}$ and is well separated from the hole. 
Numerical integration of Eqs. (22) using the 2.5 post-Newtonian equations of motion for spinning bodies ( [7] and references therein) yields results comparable to those in Tables [III].

\section{DISCUSSION}

Having obtained the leading-order changes in a black hole's mass and spin during inspiral [see Eqs. (14)], we must check whether these changes exceed the ambiguities inherent in the definitions of mass and spin [9]. Denote by $\delta M$ and $\delta S$ the magnitudes of the mass and spin ambiguities. From Eqs. (1.8) in [9],

$$
\delta M \sim \frac{M L^{2}}{\mathcal{R}^{2}}, \quad \delta S \sim \frac{M^{3} L}{\mathcal{R}^{2}},
$$

where $M$ and $L$ are the mass and size of the (isolated) body in question, and $\mathcal{R}$ is the

external universe's radius of curvature. For a black hole in a binary, say BH1, $L \sim M_{1}$ and $\mathcal{R}^{2} \sim b^{3} / M_{2}$. This implies

$$
\frac{\delta M_{1}}{M_{1}} \sim \frac{\delta S_{1}}{M_{1}^{2}} \sim \eta \frac{M_{1}}{M}\left(\frac{M}{b}\right)^{3}
$$

From Eqs. (14), the changes $\Delta M_{1}$ and $\Delta S_{1}$ from infinite separation to separation $b$ are

$$
\frac{\Delta M_{1}}{M_{1}} \sim \eta\left(\frac{M_{1}}{M}\right)^{2}\left(\frac{M}{b}\right)^{7 / 2}, \quad \frac{\Delta S_{1}}{M_{1}^{2}} \sim \eta \frac{M_{1}}{M}\left(\frac{M}{b}\right)^{2} .
$$

So, at separation $b$, we have

$$
\frac{\Delta M_{1}}{\delta M_{1}} \sim \frac{M_{1}}{M}\left(\frac{M}{b}\right)^{1 / 2}, \quad \frac{\Delta S_{1}}{\delta S_{1}} \sim \frac{b}{M} .
$$

We conclude that $\left|\Delta S_{1}\right|$ exceeds the ambiguity $\delta S_{1}$ in the definition of spin, but $\left|\Delta M_{1}\right|$ does not rise above $\delta M_{1}$. Note that the concept of tidal work is unambiguous [10 [12].

When analyzing initial data that contain a black hole and represent the interface between inspiral and merger, one can define and calculate the hole's mass and spin in different ways, giving different answers corresponding to the ambiguities $\delta M$ and $\delta S$ discussed above. Since $\delta M$ is larger than $|\Delta M|$, the hole's mass can be considered constant during inspiral to the same level of accuracy as used in defining mass. On the other hand, $|\Delta S|$ exceeds $\delta S$, so 
the hole's spin cannot be considered constant; however, as Table \indicates, the changes in spin are small during inspiral.

The results of this work - in particular, Eqs. (14) and (22) - can be used to relate the spin and horizon area of a black hole in a particular initial data set to the spin and horizon area the hole had when infinitely separated from its companion.

\section{ACKNOWLEDGMENTS}

I am grateful to Kip Thorne for helpful discussions and advice, and to Scott Hughes for useful comments on the manuscript. This research was supported in part by NSF grant PHY-0099568 and NASA grant NAG5-10707. 


\section{REFERENCES}

[1] S.F.P. Zwart and S.L.W. McMillan, Astrophys. J. 528, L17 (2000); astro-ph/9910061.

[2] H. Tagoshi, S. Mano, and E. Takasugi, Prog. Theor. Phys. 98, 829 (1997); grqc/9711072.

[3] S.A. Hughes, gr-qc/0104041.

[4] K. Alvi, Phys. Rev. D 61, 124013 (2000); gr-qc/9912113.

[5] R.H. Price and J.T. Whelan, gr-qc/0107029.

[6] L. Blanchet et al., Phys. Rev. Lett. 74, 3515 (1995); gr-qc/9501027.

[7] L.E. Kidder, Phys. Rev. D 52, 821 (1995); gr-qc/9506022.

[8] K.S. Thorne, Astrophys. J. 191, 507 (1974).

[9] K.S. Thorne and J.B. Hartle, Phys. Rev. D 31, 1815 (1985).

[10] P. Purdue, Phys. Rev. D 60, 104054 (1999); gr-qc/9901086.

[11] I.S. Booth and J.D.E. Creighton, Phys. Rev. D 62, 067503 (2000); gr-qc/0003038.

[12] M. Favata, Phys. Rev. D 63, 064013 (2001); gr-qc/0008061.

[13] Black Holes: The Membrane Paradigm, edited by K.S. Thorne, R.H. Price, and D.A. Macdonald (Yale University Press, New Haven, 1986).

[14] S.W. Hawking and J.B. Hartle, Commun. Math. Phys. 27, 283 (1972).

[15] S.A. Teukolsky, Astrophys. J. 185, 635 (1973).

[16] S.A. Teukolsky and W.H. Press, Astrophys. J. 193, 443 (1974).

[17] B. Carter, in General Relativity: An Einstein Centenary Survey, edited by S.W. Hawking and W. Israel (Cambridge University Press, New York, 1979), p. 294.

[18] J.N. Goldberg et al., J. Math. Phys. 8, 2155 (1967). 
[19] S.A. Teukolsky, Ph.D. thesis, California Institute of Technology, 1973 (unpublished).

[20] J.B. Hartle, Phys. Rev. D 8, 1010 (1973).

[21] J.B. Hartle, Phys. Rev. D 9, 2749 (1974).

[22] C.W. Misner, K.S. Thorne, and J.A. Wheeler, Gravitation (Freeman, San Francisco, 1973).

[23] P.C. Peters and J. Mathews, Phys. Rev. 131, 435 (1963).

[24] P.C. Peters, Phys. Rev. 136, B1224 (1964).

[25] E. Poisson and M. Sasaki, Phys. Rev. D 51, 5753 (1995); gr-qc/9412027.

[26] T.A. Apostolatos, C. Cutler, G.J. Sussman, and K.S. Thorne, Phys. Rev. D 49, 6274 (1994). 